\title{
Communicable Diseases Report, NSW, November and December 2010
}

\section{Communicable Diseases Branch NSW Department of Health}

For updated information, including data and facts on specific diseases, visit www.health.nsw.gov.au and click on Public Health and then Infectious Diseases. The communicable diseases site is available at: http://www.health.nsw.gov.au/publichealth/ infectious/index.asp.
Figure 1 and Tables 1 and 2 show reports of communicable diseases received through to the end of November and December 2010 in New South Wales (NSW).

\section{Enteric infections \\ Outbreaks of foodborne disease}

Nine outbreaks of suspected foodborne disease were investigated in November and December 2010. Stool specimens were collected and tested in two of these outbreaks and Salmonella Typhimurium was identified. One of these outbreaks was linked to salmon patties made with raw eggs, however no leftovers were available for testing and samples from the premises were negative. In the second outbreak the local public health unit (PHU) identified $S$. Typhimurium in two people who attended a conference. Through their investigation, the local PHU identified a further five possible cases. However, as detailed menu information was not collected and no samples were taken from the venue the food vehicle remains unknown.

Another outbreak was identified through three separate reports to the NSW Food Authority about a bakery and was found to be linked to the consumption of pork rolls. The eight affected people did not submit stool samples but food and environmental samples taken from the premises were all positive for $S$. Typhimurium.

In the remaining six outbreaks, none of the cases submitted a stool specimen for testing so the causative pathogen of the outbreaks could not be identified.

\section{Outbreaks of gastroenteritis in institutional settings}

During November and December, 43 outbreaks of gastroenteritis in institutions were reported, affecting 539 people. Twenty-three outbreaks occurred in child care centres, 14 in aged care facilities, five in hospitals, and one in a mental health facility. All outbreaks appeared to have been caused by person-to-person spread of a viral illness. In 22 outbreaks $(51 \%)$ one or more stool specimens were collected from cases; in six of these outbreaks $(27 \%)$ norovirus was detected, and in five (23\%) stool specimens tested positive for rotavirus. The remaining 11 outbreaks had negative test results. Viral gastroenteritis tends to peak in winter with around 15 outbreaks per week; over the past 5 years in November and December the average number of outbreaks has been 47 .

\section{Respiratory and other infections Influenza}

During November and December influenza activity was low in NSW, as measured by the number of patients who presented to 56 of the state's largest emergency departments with influenza-like-illness. There were 147 emergency department presentations of patients with influenza-like illness (1.0 per 1000 presentations) for November and 129 presentations ( 0.7 per 1000 presentations) for December.

The number of patients who tested positive for influenza at diagnostic laboratories was slightly above the usual level for this time of year. There were 41 cases of laboratoryconfirmed influenza (including 18 of pandemic (H1N1) 2009) reported in November. Of these $12 \%$ were aged $0-5$ years, 20\% were aged 5-9 years and 46\% were aged 15-49 years. In December, 46 cases were reported (including 37 of pandemic (H1N1) 2009). Of these, 15\% were aged $0-5$ years, $15 \%$ were aged $5-9$ years and $50 \%$ were aged 15-49 years.

For a more detailed report on respiratory activity in NSW see: http://www.health.nsw.gov.au/PublicHealth/ Infectious/influenza_reports.asp

\section{Vaccine-preventable diseases Meningococcal disease}

Nine cases of meningococcal disease were reported in NSW in November and December (12 cases were reported in the same period in 2009). The ages of the affected people 
ranged from 3 to 70 years (three cases were children aged less than 5 years). One case (in an unvaccinated adult) was caused by serogroup $\mathrm{C}$, for which there is a vaccine. Six cases were caused by serogroup B, one case by serogroup W135, and one case by serogroup Y.

In 2010, 73 cases of meningococcal disease were reported in NSW (including five deaths, one an infant aged 0-4 years) compared to 92 cases in 2009 (including four deaths in adults).

A free vaccine for serogroup $\mathrm{C}$ meningoccocal disease is available for infants at 12 months of age. Consequently, serogroup $\mathrm{C}$ meningococcal disease is now mainly seen in adults and in unimmunised children. In NSW in 2010, $82 \%$ of cases of meningococcal disease (where the serogroup was known) were caused by serogroup B, for which there is no vaccine.

\section{Pertussis (whooping cough)}

During November and December, 3450 cases of pertussis were reported in NSW. Over 20000 cases of pertussis were reported during 2008 and 2009. Case reports declined to a low in April 2010 (with 314 cases reported), but since then have increased, with 1860 cases reported in November and 1590 cases in December. The number of reported cases was highest in children aged 5-9 years and 10-14 years. In total, 9244 cases were reported in 2010 compared with 12577 in 2009.

A free vaccine is recommended for infants at 2, 4 and 6 months of age although the first dose can be given as early as 6 weeks of age. A booster dose is recommended at 4 years but this can be given as early as 3 years and 6 months of age. Immunisation reduces the risk of infection, however the vaccine does not provide lifelong protection and re-infection can occur. Because pertussis immunity wanes over time, many older children and adults are susceptible to infection and can be the source of new infections in infants. For a limited time, free pertussis (dTpa) vaccine is available for all new parents, grandparents and any other adults who will regularly care for infants less than 12 months of age. Free vaccine boosters are also provided in high school as part of the NSW SchoolBased Vaccination Program.

\section{Sexually transmissible infections Syphilis}

There was a decrease of approximately $30 \%$ in infectious syphilis notifications for NSW in 2010 compared to 2009. A total of 379 cases of infectious syphilis were reported in NSW up until the end of December 2010 compared to 533 cases notified during 2009. The majority of notifications occurred in males aged between 20 and 50 years of age, which is consistent with previous trends.

Syphilis is a highly infectious sexually transmitted disease that is spread through vaginal, anal or oral sex through skin-to-skin contact. Syphilis is highly contagious during the primary and secondary stages when the sore or rash is present. Those most at risk include men who have sex with men, people with HIV/AIDS, and people living in Aboriginal communities that are remote or have poor access to health care services.

\section{Lymphogranuloma venereum (LGV)}

An increase in lymphogranuloma venereum (LGV) notifications was reported in NSW in 2010. A total of 50 cases were reported to NSW Health from January to October 2010. The increase may have been due in part to increased screening and case detection following alerts to local clinicians. The number of reports dropped in November and December 2010, with only three cases reported.

LGV is a sexually transmitted infection. It is caused by a rare, severe strain of chlamydia which generally causes more severe symptoms than chlamydia. Around 3-30 days after exposure, a small painless lump or sore appears on or in the penis, rectum, vagina, cervix or mouth. The initial lesion heals after a few days and most people are not aware of it. Over the next 2-6 weeks the infection spreads to the local lymph glands usually in the groin or inside the pelvis. People may also have fever, chills, weight loss, feel generally unwell or have sore muscles and joints. Where the infection is around the rectum there can be a discharge of blood, pus or mucus from the anus, a painful urgent feeling of needing to pass a bowel motion but being unable to do so, diarrhoea or constipation, and lower abdominal pain. LGV is spread through unprotected vaginal, anal or oral sexual contact. It can also be spread through sharing of sex toys between partners. 
Figure 1. Reports of selected communicable diseases, NSW, January 2004 to December 2010, by month of onset. Preliminary data: case counts in recent months may increase because of reporting delays. Laboratory-confirmed cases only, except for measles, meningococcal disease and pertussis. BFV, Barmah Forest virus infection; RRV, Ross River virus infections; lab conf, laboratory confirmed; Men Gp C and Gp B, meningococcal disease due to serogroup C and serogroup B infection; other/unk, other or unknown serogroups.

NB: Multiple series in graphs are stacked, except gastroenteritis outbreaks.

NB: Outbreaks are more likely to be reported by nursing homes \& hospitals than by other institutions.

\begin{tabular}{|lr|}
\hline NSW Population \\
Male & $50 \%$ \\
$<5 \mathrm{y}$ & $7 \%$ \\
$5-24 \mathrm{y}$ & $27 \%$ \\
$25-64 \mathrm{y}$ & $53 \%$ \\
$65+\mathrm{y}$ & $13 \%$ \\
\hline
\end{tabular}

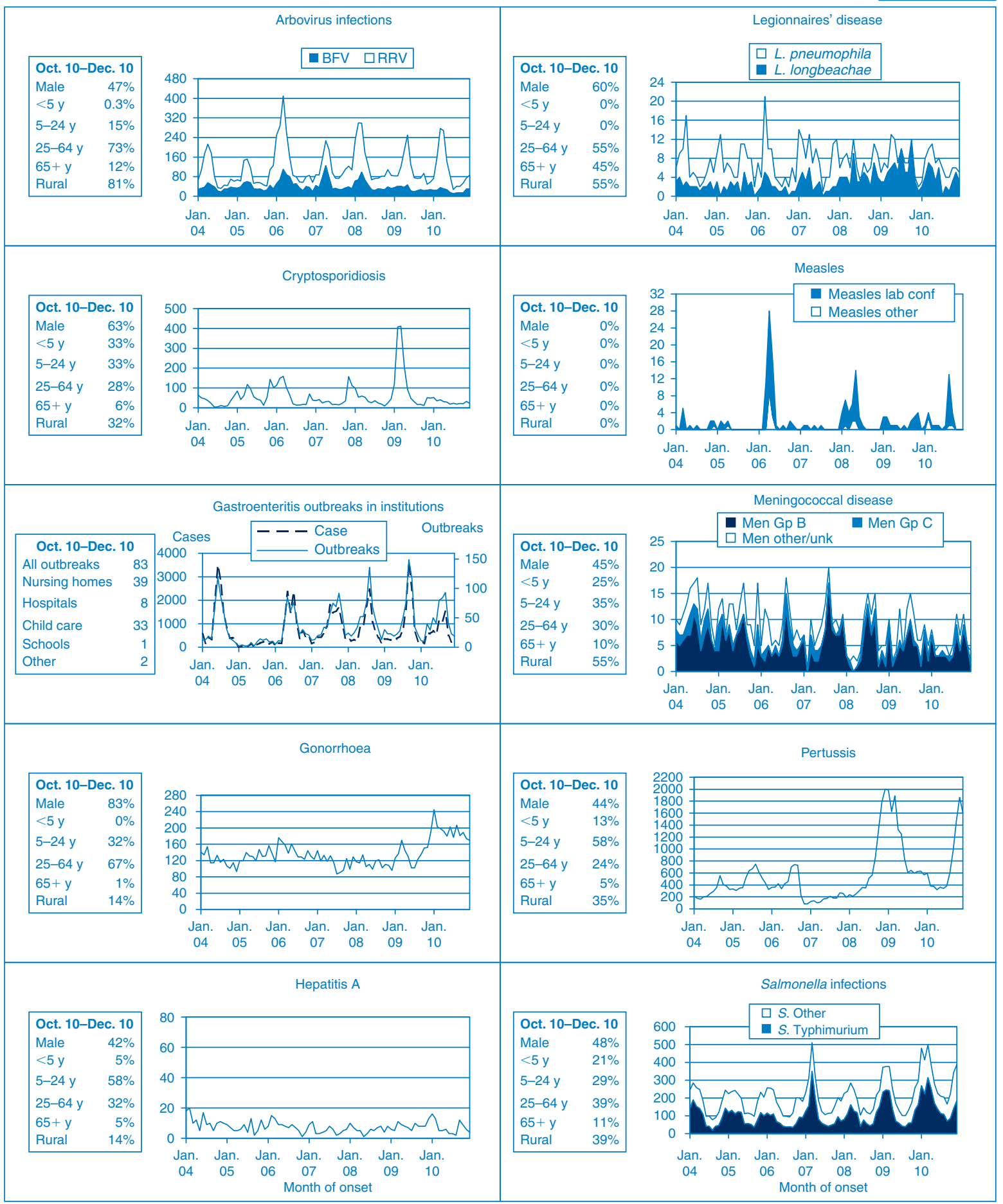




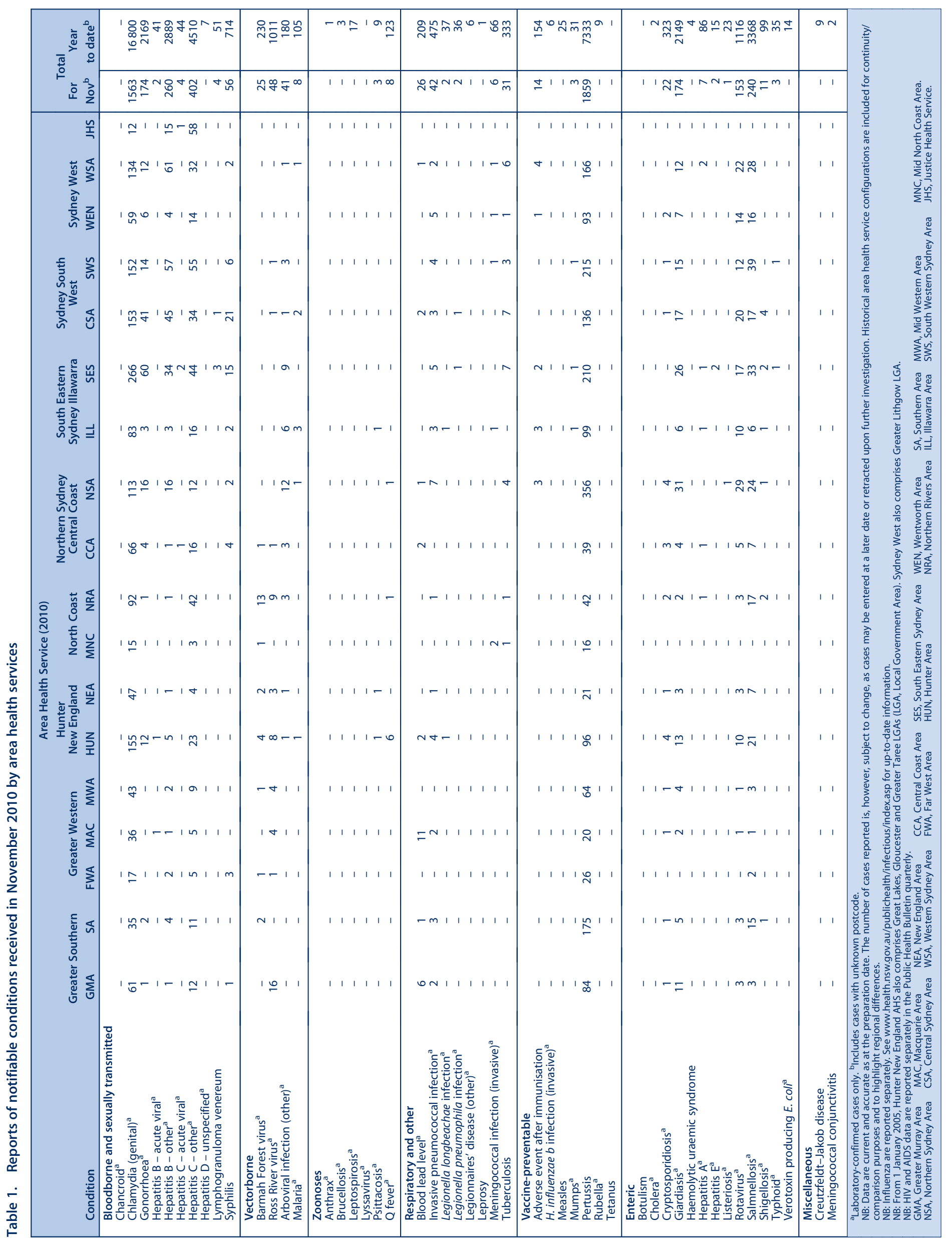




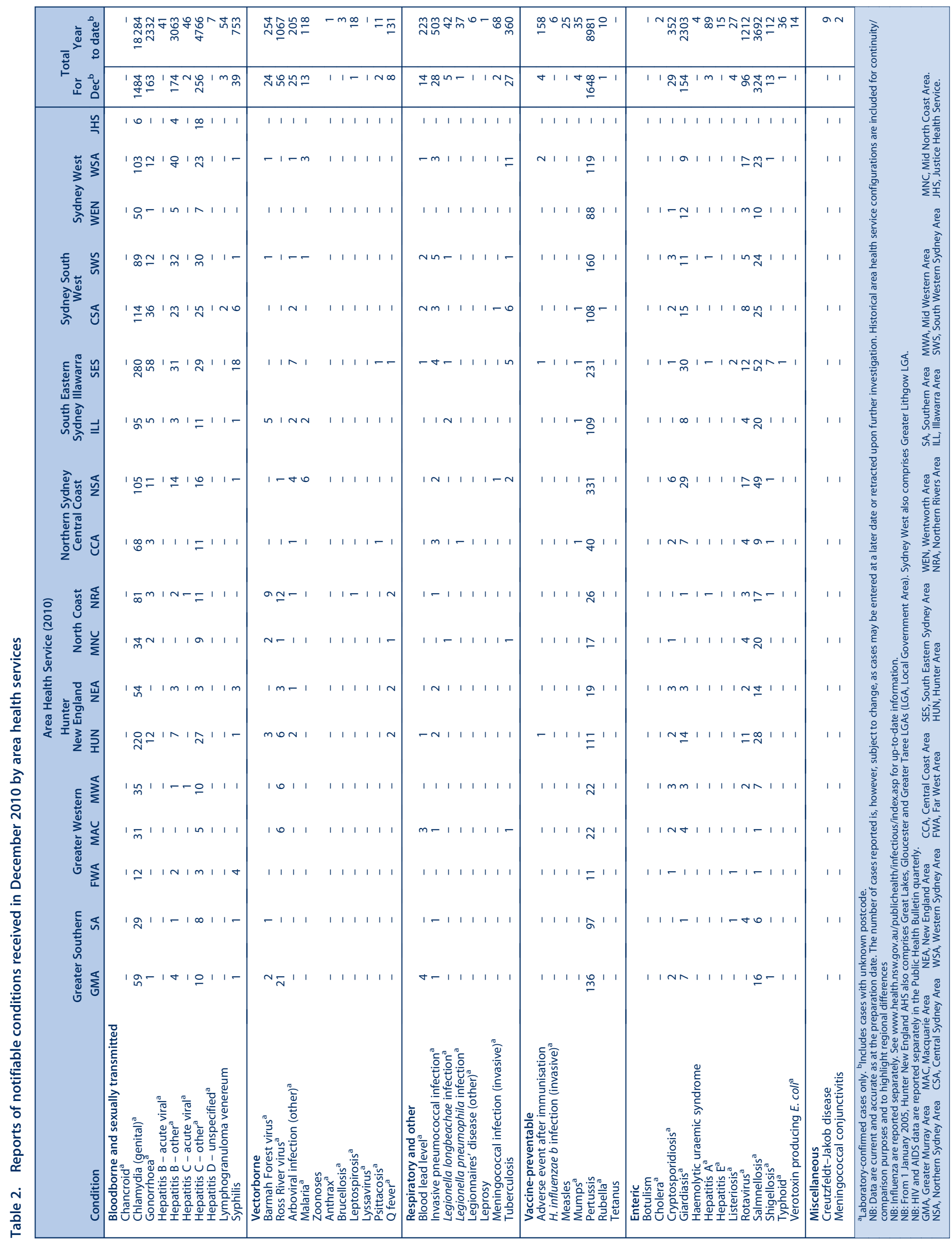

